\title{
Jones-matrix images corresponding to networks of biological crystals for diagnostics and classification of their optical properties
}

\author{
Yu. O. Ushenko, V. O. Balanetska, O. P. Angelsky \\ ${ }^{1}$ Chernivtsi National University \\ 2, Kotsyubinsky vul., 58012 Chernivtsi, Ukraine
}

\begin{abstract}
Performed in this work is a complex statistical, correlation and fractal analysis of coordinate distributions for Jones-matrix elements describing birefringent networks observed in the main types of human amino acids. Determined are the values and ranges for changing the statistical, correlation and spectral moments of the 1-st to 4-th orders that characterize Jones-matrix images of these biological polycrystalline protein structures. We have ascertained objective criteria for classification and differentiation of optical properties inherent to polycrystalline networks of amino acids with different types of spatial symmetry - dendrite, spherolite and cluster ones.
\end{abstract}

Keywords: laser, polarization, birefringence, Jones matrix, amino acid, statistical moment, autocorrelation, power spectrum.

Manuscript received 30.08.11; accepted for publication 14.09.11; published online 21.09.11.

\section{Introduction}

Among the methods for optical diagnostics of biological layers, the most widely spread are those of laser polarimetric diagnostics aimed at optical-anisotropical structure inherent to human tissues [1 - 31]. The main "information product" of these methods is obtaining the coordinate distributions for elements of Mueller and Jones matrixes corresponding to biological tissues (BT) [1 - 5] with the following statistical (statistical moments of the first to fourth orders [5, 6, 10, 14, 19, 25, 26, 30]), correlation (auto- and mutual-correlation functions [12, 17, 18, 21, 26]), fractal (fractal dimensionalities [5, 6, 25]), singular (distributions of amounts of linear and circularly polarized states), wavelet (sets of wavelet coefficients for various scales of biological crystals [22, 28]) analyses. As a result, one can determine interrelations between a set of these parameters and distributions of optical axis directions as well as the birefringence value inherent to networks of optically single-axis protein (myosin, collagen, elastin, etc.) fibrils in optically-anisotropic component of BT layer. Being based on this approach, a large amount of methods for diagnostics and differentiation of pathological changes in BT structure that are related with their degenerative- dystrophic as well as oncological changes [4 - 6, 12, 19, 20-22, 27, 29, 31]. The following progress in diagnostical possibilities of laser polarimetry can be related with account of anisotropy typical not only to fibrillar networks but to more deep level of their organization - structure of polycrystalline amino acids.

Our work is aimed at searching the possibilities for diagnostics and classification of optical properties inherent to polycrystalline networks of the main types of human amino acids via determination of coordinate distributions corresponding to Jones-matrix elements with the following statistical, correlation and fractal analyses of these distributions.

\section{Main analytical relations}

As a base for modeling the optical properties of polycrystalline networks corresponding to main types of amino acids in biological liquids, we took the following conceptions developed for optically-anisotropic protein fibrils [1-4, 7, 9, 14, 16, 23-27, 30]:

- $\quad$ separate (partial) amino acid crystals are optically single-axis and birefringent;

- optical properties of a partial crystal is exhaustively full described with the Jones operator [5] 


$$
\{J\}=\left\|\begin{array}{ll}
J_{11} & J_{12} \\
J_{21} & J_{22}
\end{array}\right\|=\left\|\begin{array}{cc}
\cos ^{2} \rho+\sin ^{2} \rho \exp (-i \delta) ; & \cos \rho \sin \rho[1-\exp (-i \delta)] ; \\
\cos \rho \sin \rho[1-\exp (-i \delta)] ; & \sin ^{2} \rho+\cos ^{2} \rho \exp (-i \delta) ;
\end{array}\right\| .
$$

Here, $\rho$ is the direction of the optical axis; $\delta=2 \pi / \lambda \Delta n d-$ phase shift between orthogonal components $E_{x}$ and $E_{y}$ of the amplitude of illuminating laser wave with the wavelength $\lambda ; \Delta n$ - birefringence index for the crystal with geometric dimension $d$.

The Jones matrix $\{\mathfrak{J}\}$ of a flat layer corresponding to polycrystalline network can be defined as a sum of operators $\{J\}$ for $K$ separate amino acid crystals [5, $11,30]$

$$
\{\mathfrak{J}\}=\sum_{l=1}^{K}\{J\}_{l},
$$

It can be shown that for $Q$ sequentially placed flat layers the net Jones matrix is defined with the expression $\{\mathfrak{N}\}=\prod_{q=1}^{Q}\{\mathfrak{J}\}_{q}=\{\mathfrak{I}\}_{Q} \times\{\mathfrak{J}\}_{Q-1} \times \ldots \times\{\mathfrak{I}\}_{2} \times\{\mathfrak{I}\}_{1}$.

In an expanded version, the matrix elements $\aleph_{i k}$ possess a cumbersome analytical look. Therefore, to consider the structure of generalized Jones matrix $\{\boldsymbol{\aleph}\}$ in more convenient manner, we limit ourselves (without losses in fullness of analysis) by the case of bilayer $(Q=2)$ polycrystalline network. With account of this approximation as well as ... look of dependences (relation (1)) for matrix elements $J_{i k}(\rho, \delta)$, it is possible to show that the elements of generalized Jones matrix can be expressed with the following dependences $\aleph_{i k}=R_{i k}(\rho)+T_{i k}(\rho) \exp (-i \delta)+U_{i k}(\rho) \exp (-2 i \delta)$. (4)

Here, $R_{i k} ; T_{i k} ; U_{i k}$ are the coefficients expressed via quasi-harmonic functionals ( " $\left.\cos ^{2} " ; " \sin ^{2} " ; " \cos -\sin "\right)$ from coordinate changes in orientations of optical axis $\rho(x, y)$

$$
\begin{aligned}
& \left\{\begin{array}{l}
R_{11}=\cos ^{2} \rho \cos ^{2}(\rho+\Delta \rho)+(0,5)^{4} \sin 2 \rho \sin 2(\rho+\Delta \rho) ; \\
T_{11}=\left[\cos ^{2} \rho \sin ^{2}(\rho+\Delta \rho)+\sin ^{2} \rho \cos ^{2}(\rho+\Delta \rho)-2 \sin 2 \rho \sin 2(\rho+\Delta \rho)\right] \exp (-i \delta) ; \\
U_{11}=\left[\sin ^{2} \rho \sin ^{2}(\rho+\Delta \rho)+2 \sin 2 \rho \sin 2(\rho+\Delta \rho)\right] \exp (-2 i \delta) .
\end{array}\right. \\
& \left\{\begin{array}{l}
R_{12 ; 21}=0,25\left[\cos ^{2} \rho \sin 2(\rho+\Delta \rho)+\sin 2 \rho \cos ^{2}(\rho+\Delta \rho)\right] \\
T_{12 ; 21}=0,25\left[\sin 2 \rho \cos ^{2}(\rho+\Delta \rho)+\sin ^{2} \rho \sin 2(\rho+\Delta \rho)\right] \exp (-i \delta) ; \\
U_{12 ; 21}=0,25\left[\sin ^{2} \rho \sin 2(\rho+\Delta \rho)+\sin 2 \rho \cos ^{2} \rho\right] \exp (-i 2 \delta) .
\end{array}\right. \\
& \left\{\begin{array}{l}
R_{22}=\sin ^{2} \rho \sin ^{2}(\rho+\Delta \rho)+(0,5)^{4} \sin 2 \rho \sin 2(\rho+\Delta \rho) ; \\
T_{22}=\left[\sin ^{2} \rho \cos ^{2}(\rho+\Delta \rho)+\cos ^{2} \rho \sin ^{2}(\rho+\Delta \rho)-2 \sin 2 \rho \sin 2(\rho+\Delta \rho)\right] \exp (-i \delta) ; \\
U_{22}=\left[\cos ^{2} \rho \cos ^{2}(\rho+\Delta \rho)+2 \sin 2 \rho \sin 2(\rho+\Delta \rho)\right] \exp (-2 i \delta) .
\end{array}\right.
\end{aligned}
$$


As a base for analyzing the coordinate structure of $\left(\aleph_{i k}\right)_{j}$ distributions, we took the autocorrelation method with using the function $[12,21,26]$

$G(\Delta x)=\frac{1}{X_{0}} \int_{1}^{X_{0}}\left[\aleph_{i k}(x)\right]\left[\aleph_{i k}(x-\Delta x)\right] d x$.

Here, $\Delta x$ is the "step" of changing the coordinates $x=1 \div X_{0}$.

As parameters that characterize the dependences $G(\Delta x)$, we chose the set of correlation moments of the 1-st to 4-th orders $K_{l=1 ; 2 ; 3 ; 4}$ that are defined like to relations (8).

Estimating the degree of self-similarity or repeatability for different geometric $(d)$ scales of the structure inherent to coordinate distributions of elements $\aleph_{i k}$ corresponding to the Jones matrix of polycrystalline networks was performed by calculation of logarithmic dependences for power spectra $\log J\left(\aleph_{i k}\right)-\log \left(d^{-1}\right)$ that were approximated using the least-squares method to the curves $\Phi(\eta)$. For the straight parts of the curves $\Phi(\eta)$, determined are the slope angles $\eta_{i}$ and calculated are the values of fractal dimensionalities for the sets of $\aleph_{i k}$ values by using the relations $[5,6,11,25]$

$D_{i}(g)=3-\operatorname{tg}_{i}$.

Classification of coordinate distributions for matrix elements $\aleph_{i k}(x, y)$ was carried out in accord with the criteria offered in [5].

If the value of the slope angle $\eta=$ const in the dependences $\Phi(\eta)$ for 2 or 3 decades of changing the sizes $d$, then the distributions $\aleph_{i k}(x, y)$ are fractal. Under condition that several constant slope angles are available in the curve $\Phi(\eta)$, the sets $\aleph_{i k}(x, y)$ are multifractal. When no stable slope angles are available over the whole interval of changing the sizes $d$, the sets $\aleph_{i k}(x, y)$ are considered as random.

To make this comparative analysis of $\log J\left(\aleph_{i k}\right)-\log \left(d^{-1}\right)$ dependences more objective, let us introduce the conception of spectral moments from the 1-st to 4-th orders $S_{j=1 ; 2 ; 3 ; 4}$ - the relation (8).

\section{Computer modeling the Jones-matrix images corresponding to multi-layer polycrystalline network}

With the aim to obtain objective criteria for diagnostics of the structure inherent to these multilayer birefringent networks, we have performed computer modeling the dependence of coordinate distributions for elements of the Jones matrix $\aleph_{i k}(m \times n)$ on changes of the orientation-phase structure of flat layers as well as their number.

Our computer modeling was based on the following assumptions:

- multi-layer network is considered as a set of planar sequentially located networks of birefringent cylinders with parallel optical axes;

- each planar layer consists of $Q=20$ parallel densely-packed cylinders of the length $\mathrm{L}=800 \mu \mathrm{m}$ and diameter $\otimes=40 \mu \mathrm{m}$ and birefringent index $\Delta n=1.75 \times 10^{-2}$;

- within the limits of cross-section for a partial cylinder, the full period $(0 \div 2 \pi)$ of the phase change $\delta(r=0 \div \otimes)=2 \pi / \lambda \Delta n l(r=0 \div \otimes)$ is realized;

- multi-layer network (the amount of layers is $q$ ) is formed via sequential arrangement of flat layers turned to each other by the angle $\gamma_{q}=\gamma_{q-1}+\pi / q$.

To calculate coordinate distributions for Jonesmatrix elements $\aleph_{i k}$, the plane XOY where placed is a partial flat layer of oriented birefringent cylinders was considered as a two-dimensional array of discrete square pixels of the linear size $d=2 \mu \mathrm{m}$. In other words, there formed is a 2D analyzed array $m \times n=400$ pix $\times 400$ pix in the plane of this partial layer, within the framework of which one can calculate the Jones-matrix images $\aleph_{i k}(m \times n)$.

Depicted in Fig. 1 are the results of our computer modeling the coordinate structure for the Jones-matrix image of one of the $\aleph_{22}(m \times n)$ elements corresponding to 6-layer birefringent polycrystalline network and its statistical, correlation and fractal parameters.

The analysis of results obtained using computer modeling the structure of Jones-matrix images describing the 6-layer birefringent polycrystalline network has confirmed adequacy of our analytical modeling (relations (1) to (7)) and shown that:

- coordinate distribution $\aleph_{22}^{z=6}(m \times n)$ is "qausi-regular" with sufficiently equiprobable distribution $H\left(\aleph_{22}\right)$ within the limits of values 0 to 1 ;

- dependence of the autocorrelation function $G_{22}(\Delta m, \Delta n)$ for coordinate distribution $\aleph_{22}(m \times n)$ drops monotonically with different-frequency oscillations of intrinsic values;

- logarithmic dependences $\log J\left(\aleph_{i k}\right)-\log \left(d^{-1}\right)$ for the power spectra corresponding to the coordinate distribution of Jones-matrix elements $\aleph_{22}(m \times n)$ possess a stable slope angle characterizing the approximating curve $\Phi(\eta)$. 


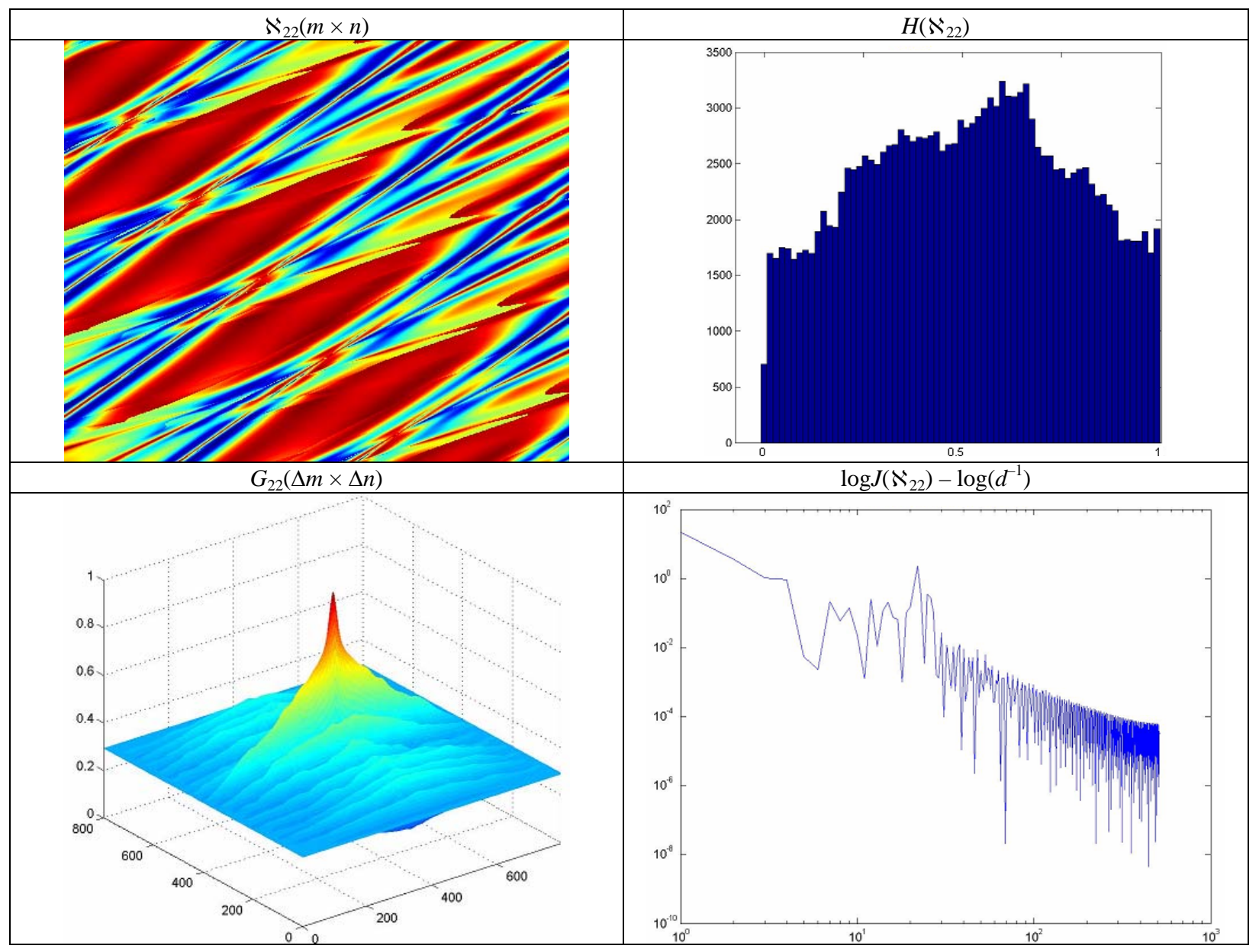

Fig. 1. Coordinate $\aleph_{22}(m \times n)$, probability $H\left(\aleph_{22}\right)$, correlation $G_{22}(\Delta m \times \Delta n)$ and self-similar $\log J\left(\aleph_{22}\right)-\log \left(d^{-1}\right)$ structures of the Jones-matrix element $\aleph_{22}$ corresponding to the multi-layer $(q=6)$ birefringent network.

The obtained results can be related with multiple contribution of "orientation" $(\rho)$ and "phase" $(\delta)$ parameters inherent to networks of partial crystals to values of Jones-matrix elements $\left\{\aleph_{i k}\right\}$ describing this polycrystalline network. So, the following values take place for these six flat layers: $\rho=0 ; \rho=0+0,1 \pi$; $\rho=0+0,2 \pi ; \ldots ; \rho=0+0,5 \pi$ and $\delta=0 \div 2 \pi ; 2 \delta ; 3 \delta$; $\ldots ; 5 \delta$. Therefore, the coordinate distribution of the $\aleph_{22}(m \times n)$ element in the generalized Jones matrix operator as well as respective autocorrelation function $G_{22}(\Delta m, \Delta n)$ is a multi-frequency dependence. Besides, the coordinate-repeated oscillations of intrinsic values corresponding to Jones-matrix images are the very cause for formation of scale-similar clusters, which results in appearance of the fractal structure in the $\aleph_{22}(m \times n)$ distribution.

From the quantitative viewpoint, transformation of Jones-matrix images $\aleph_{22}(m \times n)$ and $\aleph_{12 ; 21}(m \times n)$ describing a polycrystalline network of optically uniaxial birefringent crystals with different numbers $(q=3$; of flat layers can be illustrated with the set of statistical $(M, \sigma, A, E)$, correlation $\left(K_{i=1 ; 2 ; 3 ; 4}\right)$ and spectral $\left(S_{i=1 ; 2 ; 3 ; 4}\right)$ moments of the 1-st to 4-th orders, which are summarized in Table 1.

Table 1. Statistical, correlation and spectral moments of the 1-st to 4-th orders for $\aleph_{i k}(m \times n)$ distributions typical for a multi-layer $(q)$ polycrystalline network

\begin{tabular}{|c|c|c|c|c|}
\hline \multirow{2}{*}{$\begin{array}{c}\text { Para- } \\
\text { meters }\end{array}$} & \multicolumn{2}{|c|}{$q=3$} & \multicolumn{2}{c|}{$q=6$} \\
\cline { 2 - 5 } & $\aleph_{22}(m \times n)$ & $\aleph_{12 ; 21}(m \times n)$ & $\aleph_{22}(m \times n)$ & $\aleph_{12 ; 21}(m \times n)$ \\
\hline$M$ & 0.35 & 0.28 & 0.52 & 0.46 \\
\hline$\sigma$ & 0.15 & 0.11 & 0.18 & 0.22 \\
\hline$A$ & 0.61 & 0.55 & 0.21 & 0.13 \\
\hline$E$ & 0.34 & 0.49 & 0.11 & 0.125 \\
\hline$K_{1}$ & 0.49 & 0.46 & 0.42 & 0.44 \\
\hline$K_{2}$ & 0.044 & 0.016 & 0.072 & 0.031 \\
\hline$K_{3}$ & 2.15 & 1.89 & 0.73 & 1.08 \\
\hline$K_{4}$ & 6.36 & 5.29 & 3.23 & 2.14 \\
\hline$S_{1}$ & 0.69 & 0.86 & 0.44 & 0.48 \\
\hline$S_{2}$ & 0.28 & 0.45 & 0.085 & 0.11 \\
\hline$S_{3}$ & 2.22 & 4.61 & 1.37 & 1.16 \\
\hline$S_{4}$ & 1.89 & 2.51 & 1.19 & 135 \\
\hline
\end{tabular}




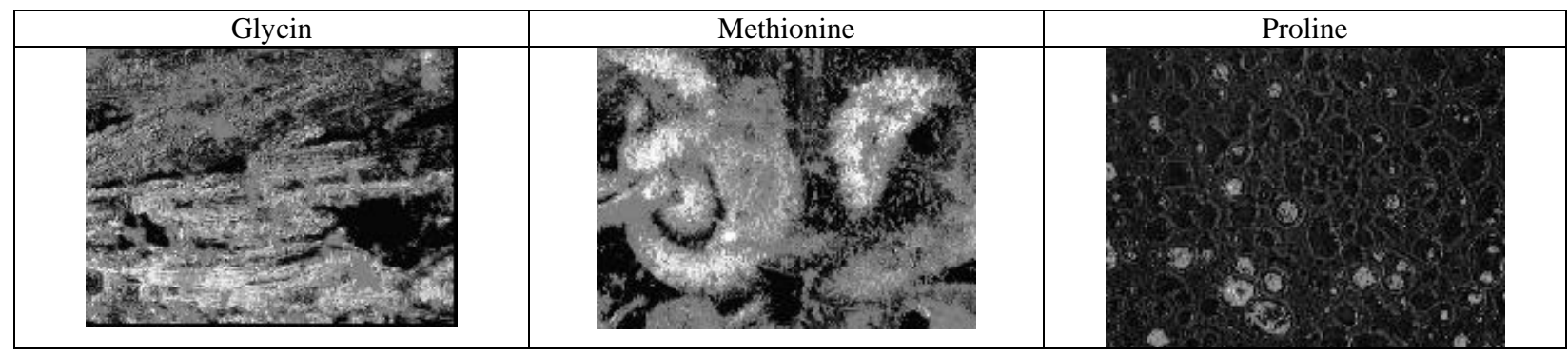

Fig. 2. Polycrystalline networks of main human amino acid types.

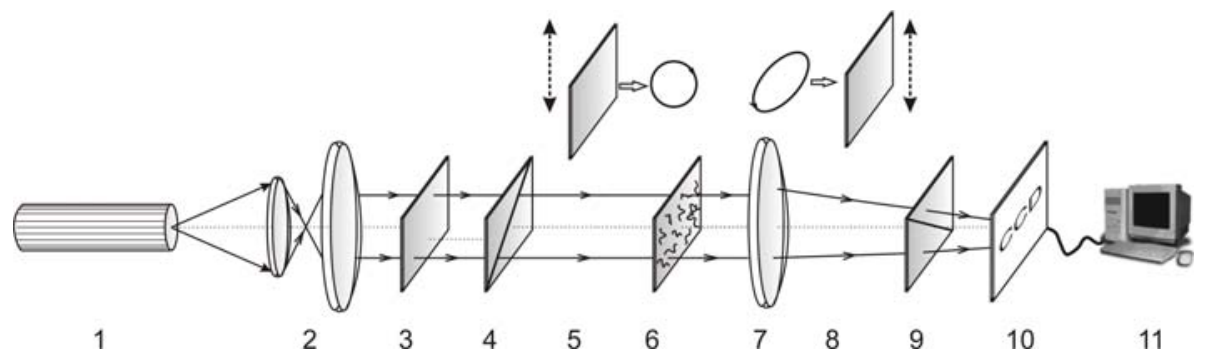

Fig. 3. Optical scheme of the polarimeter: 1 - He-Ne laser; 2 - collimator; 3 - stationary quarter-wave plate; $5,8-$ mechanically movable quarter-wave plates; 4,9 - polarizer and analyzer, respectively; 6 - object of investigation; 7 - microobjective; 10 - CCD camera; 11 - personal computer.

As seen, the whole set of statistical moments of the 1-st to 4-th orders, which characterize coordinate distributions for Jones-matrix elements, differs from zero. Second, the values of statistical parameters are individual in Jones-matrix images of different elements. In this case, the values of statistical moments of higher orders $(A, E)$ considerably exceeds the values of mean $(M)$ and dispersion $(\sigma)$. This fact is indicative of an essential difference of these coordinate distributions $\aleph_{i k}(m \times n)$ from the normal or Gaussian ones $[\ldots]$.

The set of correlation moments of the 1-st to 4-th orders that characterize the autocorrelation functions $G_{i k}(\Delta x)$ is not only different from zero but is extraordinary "sensitive" to peculiarities of the coordinate structure. The values of correlation moments of higher orders $\left(K_{i=3 ; 4}\right)$ essentially exceed by two orders of magnitude the values of mean $\left(K_{1}\right)$ and dispersion $\left(K_{2}\right)$.

The set of spectral moments of the 1-st to 4-th orders $S_{i=1 ; 2 ; 3 ; 4}$ that characterize the dependences $\log J\left(\aleph_{i k}\right)-\log \left(d^{-1}\right)$ is also characterized with individual sensitivity to coordinate self-similarity of the structure inherent to Jones-matrix images.

Our comparative analysis performed for the set of statistical $(M, \sigma, A, E)$, correlation $\left(K_{i=1 ; 2 ; 3 ; 4}\right)$ and spectral ( $\left.S_{i=1 ; 2 ; 3 ; 4}\right)$ moments of the 1-st to 4-th orders for distributions of Jones-matrix elements has shown that with increasing the number of flat layers in birefringent networks the following tendencies take place:

- mean $M$ is 1.5 - 1.7 times increased; dispersion $\sigma$ is also increased noticeably (by $1.2-2$ times); the most expressed are changes in statistical moments of higher orders $(A, E)$ - their values are 3- to 4fold decreased;

- correlation moment of the 2-nd order $\left(K_{2}\right)$ increases by $1.5-1.85$ times; correlation moments of higher orders decrease, respectively, from 2 times $\left(K_{3}\right)$ up to 3.5 times $\left(K_{4}\right)$;

- spectral moments demonstrate the following behavior: $S_{1}$ is $1.5-1.8$ times decreased; $S_{2}$ is $3-$ 4 times decreased; $S_{3}$ is $1.75-4$ times decreased; $S_{4}$ is $1.3-1.9$ times decreased.

\section{Jones-matrix images of polycrystalline networks in optically thin amino acid layers}

As investigated objects, we chose optically thin (extinction coefficient $\tau \leq 0.01$ ) polycrystalline layers of amino acids (three types), namely: glycin, methionine and proline. This choice of objects is caused by the fact that, on the one hand, these compounds are the main "building" material for formation of protein structures in biological tissues. On the other hand, the coordinate structure of these networks sharply differs in its geometric shape - dendrite-like networks of glycin, azimuth-symmetrical spherolite networks of methionine, island (cluster) networks of proline (Fig. 2). 


\begin{tabular}{|c|c|c|}
\hline$\kappa_{22}(m \times n)$ & & $\kappa_{12 ; 21}(m \times n)$ \\
\hline & & \\
\hline & & \\
\hline
\end{tabular}

Fig. 4. Coordinate $\aleph_{22 ; 12 ; 21}(m \times n)$, correlation $G_{22 ; 12 ; 21}(\Delta m \times \Delta n)$ and self-similar $\log J\left(\aleph_{22 ; 12 ; 21}\right)-\log \left(d^{-1}\right)$ structures of Jones-matrix elements corresponding to polycrystalline network of the glycin layer.

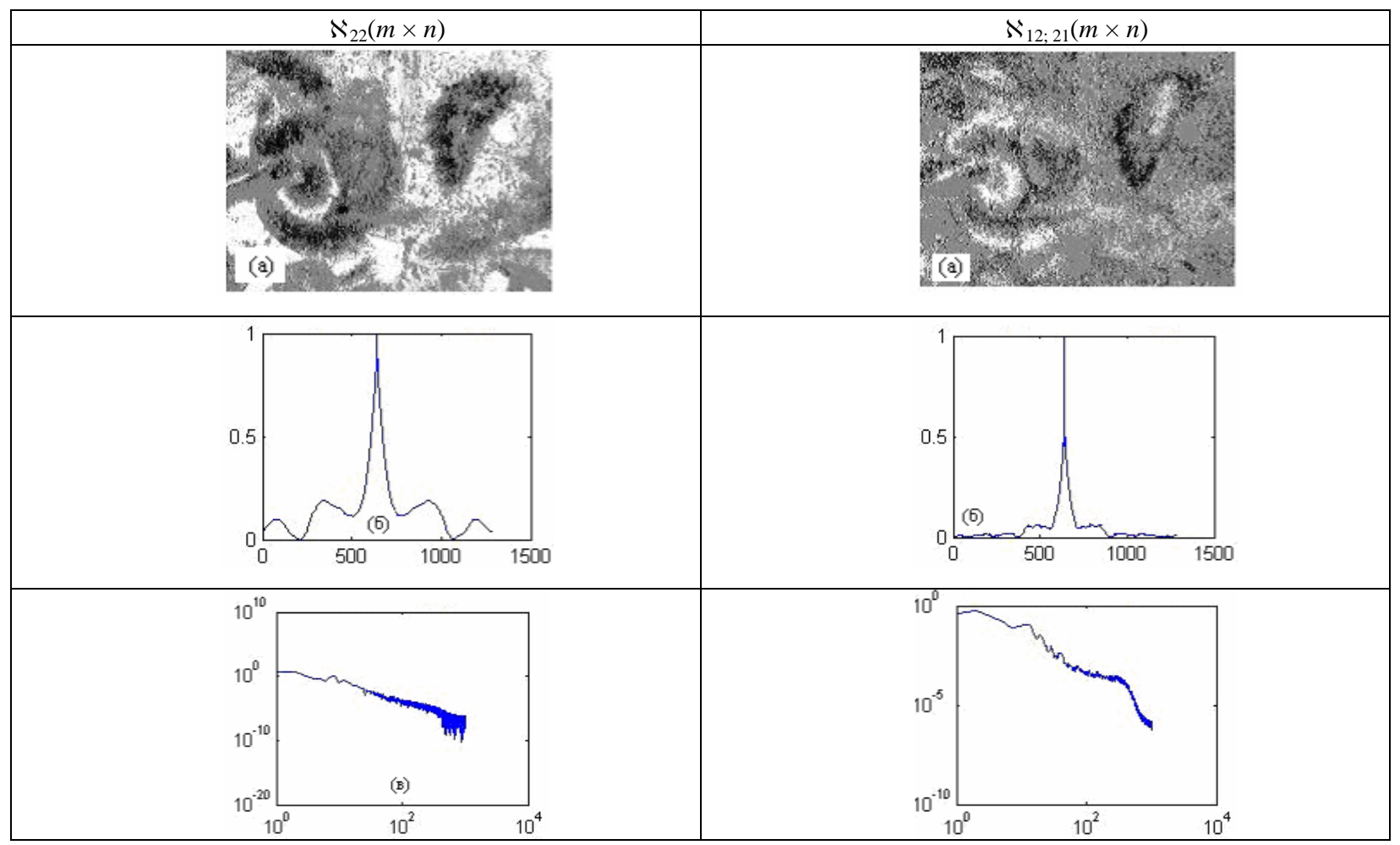

Fig. 5. Structure of Jones-matrix elements corresponding to polvcrvstalline network of the methionine laver. 


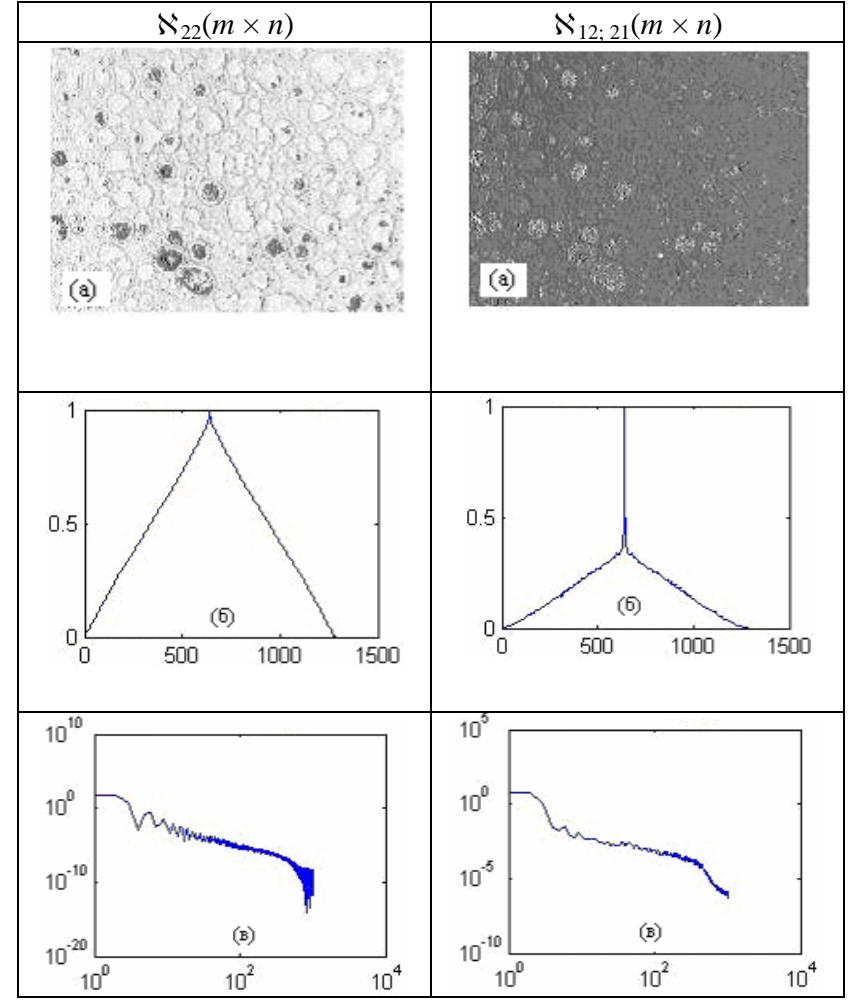

Fig. 6. Structure of Jones-matrix elements corresponding to polycrystalline network of the proline layer.

Fig. 3 shows the optical setup for measuring the coordinate distributions of Jones-matrix elements corresponding to birefringent layers.

Illumination was provided with a parallel $\left(\varnothing=10^{4} \mu \mathrm{m}\right)$ beam of a He-Ne laser $(\lambda=0.6328 \mu \mathrm{m}$, $\mathrm{W}=5.0 \mathrm{~mW})$. The polarization illuminator consists of the quarter-wave plates 3,5 and polarizer 4 , which provides formation of a laser beam with an arbitrary polarization state. Using the micro-objective 7 (magnification $4 \mathrm{x}$ ), images of biological layers were projected onto the plane of light-sensitive area (800x600 pixels) of the CCD-camera 10 that provided measurements of structural elements within the range 2 to $2000 \mu \mathrm{m}$. The analysis of laser images was performed using the polarizer 9 and quarter-wave plate 8 .

Depicted in Figs 4 to 6 is the series of experimentally measured Jones-matrix images (fragments (a)) of various elements $\aleph_{22}(m \times n)$ (left columns) and $\aleph_{12 ; 21}(m \times n) \quad$ (right columns); autocorrelation functions $G_{22}(\Delta x) ; G_{12 ; 21}(\Delta x)$ (fragments (b)); logarithmic dependences $\log J\left(\aleph_{i k}\right)-\log \left(d^{-1}\right) \quad$ (fragments (c)) of polycrystalline glycin (Fig. 4), methionine (Fig. 5) and proline (Fig. 6) layers.

The results of investigations of $\aleph_{22}(m \times n)$ and $\aleph_{12 ; 21}(m \times n)$ elements in Jones-matrix images corresponding to polycrystalline layers consisting of human main type amino acids have shown:

1. The significant influence of features inherent to orientation-phase structure in the network of amino acid partial crystals. It is confirmed by the wide range of changes $\left(0 \leq \Delta \aleph_{11} \leq 1\right)$ in intrinsic values of matrix elements corresponding to crystalline layers of all the amino acids (Figs 4 to 6, fragments (a)). There, all the coordinate distributions $\aleph_{i k}(m \times n)$ are individual for polycrystalline networks with varying geometrical structure (Figs 4 to 6, fragments (b)).

2. Autocorrelation functions $G_{11 ; 12 ; 21}(\Delta x)$ for coordinate distributions of the Jones-matrix elements corresponding to amino acid crystalline layers with expressed dendrite and spherolite geometry are decaying dependences with clearly pronounced fluctuations of intrinsic values (Figs 4 and 5, fragments (b)). The found regularity is in a good accordance with the data of model analysis (relations (1) to (7)) and data of computer modeling (Fig. 1) for the structure of respective Jonesmatrix images $\aleph_{i k}(m \times n)$. By contrast, the spatially non-oriented cluster structure of proline partial crystals does not show any fluctuations of the autocorrelation functions $G_{11 ; 12 ; 21}(\Delta x)$.

3. Sets of values for coordinate distributions $\aleph_{22}(m \times n)$ are practically fractal, since the respective approximating curves $\Phi(\eta)$ independently of the spatial symmetry type (dendrite, spherolite, cluster) inherent to birefringent networks (Fig. 3) of amino acid polycrystalline layers are characterized with a constant slope angle over the whole range of changes in geometric sizes of partial crystals (Figs 4 and 5, fragments (c)). The Jones-matrix image for the element $\aleph_{12 ; 21}(m \times n)$ is multi-fractal, since the logarithmic dependences $\log J\left(\aleph_{12 ; 21}\right)-\log \left(d^{-1}\right)$ possess two slope angles in the approximating curve $\Phi(\eta)$ (Figs 4 and 5, fragments (c)). The found self-similarity of Jones-matrix images is in a good correlation with the model data (Fig. 1) concerning the influence of coordinate-ordered changes in orientation $\rho(m \times n)$ of optical axes corresponding to partial crystals of amino acids with simultaneous multiple modulation of the phase period $\delta(m \times n)$ (relations (3) to (7)).

The results of our quantitative analysis of values and ranges for changing the statistical ( $M, \sigma, A, E$ ), correlation ( $\left.K_{i=1 ; 2 ; 3 ; 4}\right)$, spectral $\left(S_{i=1 ; 2 ; 3 ; 4}\right)$ moments of the 1-st to 4-th orders that characterize the coordinate distributions of Jones-matrix elements $\aleph_{i k}(m \times n)$ corresponding to polycrystalline layers consisting of various amino acids are summarized in Table 2.

The analysis of the obtained experimental data enabled us to ascertain satisfactory correlation with the results of computer modeling and showed that all the set 
Table 2. Statistical $(M ; \sigma ; A ; E)$, correlation $\left(K_{i=1 ; 2 ; 3 ; 4}\right)$, spectral $\left(S_{i=1 ; 2 ; 3 ; 4}\right)$ parameters of the Jones-matrix images $\aleph_{i k}(m \times n)$ corresponding to polycrystalline layers consisting of various amino acids

\begin{tabular}{|c|c|c|c|c|c|c|c|c|c|c|c|}
\hline \multicolumn{6}{|c|}{$\aleph_{22}(m \times n)$} & \multicolumn{6}{|c|}{$\aleph_{12 ; 21}(m \times n)$} \\
\hline \multicolumn{12}{|c|}{ Glycine } \\
\hline$M$ & 0.31 & $K_{1}$ & 0.44 & $S_{1}$ & 0.56 & $M$ & 0.24 & $K_{1}$ & 0.48 & $S_{1}$ & 0.53 \\
\hline$\sigma$ & 0.11 & $K_{2}$ & 0.14 & $S_{2}$ & 0.31 & $\sigma$ & 0.09 & $K_{2}$ & $0 / 14$ & $S_{2}$ & 0.14 \\
\hline$A$ & 0.86 & $K_{3}$ & 1.31 & $S_{3}$ & 0.96 & $A$ & 0.44 & $K_{3}$ & $0 / 89$ & $S_{3}$ & 0.43 \\
\hline$E$ & 0.63 & $K_{4}$ & 3.16 & $S_{4}$ & 1.43 & $E$ & 0.37 & $K_{4}$ & 1.19 & $S_{4}$ & 0.37 \\
\hline \multicolumn{12}{|c|}{ Methionine } \\
\hline$M$ & 0.46 & $K_{1}$ & 0.52 & $S_{1}$ & 0.48 & $M$ & 0.38 & $K_{1}$ & 0.51 & $S_{1}$ & 0.35 \\
\hline$\sigma$ & 0.21 & $K_{2}$ & 0.21 & $S_{2}$ & 0.23 & $\sigma$ & 0.14 & $K_{2}$ & 0.43 & $S_{2}$ & 0.11 \\
\hline$A$ & 0.35 & $K_{3}$ & 0.57 & $S_{3}$ & 0.47 & $A$ & 0.18 & $K_{3}$ & 0.54 & $S_{3}$ & 0.21 \\
\hline$E$ & 0.28 & $K_{4}$ & 1.71 & $S_{4}$ & 0.39 & $E$ & 0.12 & $K_{4}$ & 0.91 & $S_{4}$ & 0.17 \\
\hline \multicolumn{12}{|c|}{ Proline } \\
\hline$M$ & 0.65 & $K_{1}$ & 0.45 & $S_{1}$ & 0.256 & $M$ & 0.54 & $K_{1}$ & 0.48 & $S_{1}$ & 0.12 \\
\hline$\sigma$ & 0.32 & $K_{2}$ & 0.36 & $S_{2}$ & 0.13 & $\sigma$ & 0.32 & $K_{2}$ & 0.67 & $S_{2}$ & 0.05 \\
\hline$A$ & 0.12 & $K_{3}$ & 0.24 & $S_{3}$ & 0.18 & $A$ & 0.09 & $K_{3}$ & 0.16 & $S_{3}$ & 0.11 \\
\hline$E$ & 0.09 & $K_{4}$ & 0.73 & $S_{4}$ & 0.11 & $E$ & 0.04 & $K_{4}$ & 0.15 & $S_{4}$ & 0.08 \\
\hline
\end{tabular}

of statistical, correlation and spectral moments, which characterizes the coordinate distributions of Jonesmatrix elements, possesses individual set of values dependent on optical-and-geometrical parameters inherent to amino acid polycrystalline networks. For example, the change in spatial symmetry of amino acid partial crystals in the sequence "dendrite network spherolite network - cluster ensemble” is pronounced as:

- $\quad$ growth of mean $(M)$ and dispersion $(\sigma)$ and, just opposite, decrease in the values of statistical moments of the 3-rd $(A)$ and 4-th $(E)$ orders for coordinate distributions $\aleph_{22}(m \times n)$ and $\aleph_{12 ; 21}(m \times n)$;

- decay of oscillations inherent to autocorrelation functions of Jones-matrix images as well as decrease in respective values of correlation moments of higher orders $K_{3}$ and $K_{4}$ for these dependences at the background of growth typical for the correlation moment of the 2-nd order $K_{2}$;

- $\quad$ decrease in all the set of spectral moments of the 1-st to 4 -th orders $S_{i=1 ; 2 ; 3 ; 4}$ that characterize the logarithmic dependences $\log J\left(\aleph_{i k}\right)-\log \left(d^{-1}\right)$. The range of differences between the values of experimentally found statistical, correlation and spectral moments of the 1-st to 4 -th orders (Table 2) is $1.5-2$ times higher than those of computer modeling (Table 1 ).

\section{Conclusions}

1. Offered is the method of superposition for Jones matrixes to describe properties of multi-layer polycrystalline networks, and ascertained are interrelations between the orientation-phase structure of crystalline networks and the structure of Jones-matrix images.

2. It is also ascertained that Jones-matrix images of human amino acid polycrystalline layers with different types of spatial symmetry possess individual values of statistical, correlation and spectral moments of the 1-st to 4-th orders.

3. Found and grounded is the complex of criteria for Jones-matrix diagnostics and classification of optical properties typical for birefringent dendrite, spherolite and cluster polycrystalline networks of human amino acids. 


\section{References}

1. W.-F. Cheong, S. A. Prahl, A. J. Welch, "A Review of the Optical Properties of Biological Tissues," IEEE J. Quantum Electron, Vol. 26, pp. 21662185, Dec. 1990.

2. S. A. Prahl, M. Keijzer, S. L. Jacques, A. J. Welch, "A Monte Carlo model of light propagation in tissue," SPIE Proceedings of Dosimetry of Laser Radiation in Medicine and Biology, Vol. IS 5, pp. 102-111, 1989.

3. E. Wolf, "Unified theory of coherence and polarization of random electromagnetic beams," Phys. Lett. A., Vol. 312, pp. 263-267, 2003.

4. Alexander G. Ushenko and Vasilii P. Pishak, "Laser Polarimetry of Biological Tissue: Principles and Applications", in Handbook of CoherentDomain Optical Methods: Biomedical Diagnostics, Environmental and Material Science, vol. I, Valery V. Tuchin, Ed. Boston: Kluwer Academic Publishers, 2004, pp. 93-138.

5. O. V. Angelsky, A. G. Ushenko, Yu. A. Ushenko, V. P. Pishak, "Statistical and Fractal Structure of Biological Tissue Mueller Matrix Images”, in Optical Correlation Techniques and Applications, Oleg V. Angelsky, Ed. Washington: Society of Photo-Optical Instrumentation Engineers , 2007, pp. 213-266.

6. O.V. Angelsky, A.G. Ushenko, Yu.A. Ushenko, V.P. Pishak, and A.P. Peresunko, "Statistical, Correlation, and Topological Approaches in Diagnostics of the Structure and Physiological State of Birefringent Biological Tissues”, in Handbook of Photonics for Biomedical Science, Valery V. Tuchin, Ed. USA: CRC Press, 2010, pp. 21-67.

7. Alexander G. Ushenko, "Polarization structure of laser scattering fields,” Optical Engineering, vol. 34(4), pp. 1088-1093, November 1995.

8. A.G. Ushenko, "Laser diagnostics of biofractals," Quantum Electronics, vol. 29(12), pp. 1078-1084, December 1999.

9. O.V. Angel'skii, A.G. Ushenko, A.D. Arkhelyuk, S.B. Ermolenko, D.N. Burkovets, "Structure of matrices for the transformation of laser radiation by biofractals,” Quantum Electronics, vol. 29(12), pp. 1074-1077, December 1999.

10. O.V. Angel'skii, A.G. Ushenko A.D. Arheluk, S.B. Ermolenko, D. N. Burkovets, "Scattering of Laser Radiation by Multifractal Biological Structures," Optics and Spectroscopy, vol. 88(3), pp. 444-448, March 2000.

11. A.G. Ushenko, "Polarization Structure of Biospeckles and the Depolarization of Laser Radiation,” Optics and Spectroscopy, vol. 89(4), pp. 597-601, October 2000

12. A.G. Ushenko, "Stokes-correlometry of biotissues," Laser Physics, vol. 10(5), pp.12861292, May 2000.
13. A.G. Ushenko, "The Vector Structure of Laser Biospeckle Fields and Polarization Diagnostics of Collagen Skin Structures," Laser Physics, vol. 10(5), pp. 1143-1149, May 2000.

14. A.G. Ushenko, "Laser polarimetry of polarizationphase statistical moments of the object field of optically anisotropic scattering layers," Optics and Spectroscopy, vol. 91(2), pp. 313-316, February 2001.

15. A.G. Ushenko, "Polarization contrast enhancement of images of biological tissues under the conditions of multiple scattering," Optics and Spectroscopy, vol. 91(6), pp. 937-940, August 2001.

16. A.G. Ushenko, "Laser probing of biological tissues and the polarization selection of their images," Optics and Spectroscopy, vol. 91(6), pp.932-936, August 2001.

17. A.G. Ushenko, "Correlation processing and wavelet analysis of polarization images of biological tissues," Optics and Spectroscopy, vol. 91(5), pp.773-778, June 2002.

18. A.G. Ushenko, "Polarization correlometry of angular structure in the microrelief pattern or rough surfaces," Optics and spectroscopy, vol. 92(2), pp. 227-229, June 2002.

19. O.V. Angelsky, A.G. Ushenko, Ye.G. Ushenko, “2D Stokes Polarimetry of Biospeckle Tissues Images in Pre-Clinic Diagnostics of Their Pre-Cancer States," Journal of Holography and Speckle, vol. 2(1), pp. 26-33, April 2005.

20. Oleg V. Angelsky, Alexander G. Ushenko, and Yevheniya G. Ushenko, "Complex degree of mutual polarization of biological tissue coherent images for the diagnostics of their physiological state,” J. Biomed. Opt., vol. 10(6), Article ID 060502, November 2005.

21. O. V. Angelsky, A. G. Ushenko, and Ye. G. Ushenko, "Investigation of the correlation structure of biological tissue polarization images during the diagnostics of their oncological changes," Physics in Medicine and Biology, vol. 50(20), pp. 48114822, September 2005.

22. Oleg V. Angelsky, Alexander G. Ushenko, Yevheniya G. Ushenko, Yuriy Y. Tomka, "Polarization singularities of biological tissues images,” J. Biomed. Opt., vol. 11(5), Article ID 054030, September-October 2006.

23. O.G. Ushenko, S.G. Guminetsky, A.V. Motrich, "Optical properties of urine, blood plasma and pulmonary condensate of the patients with pulmovnary form of tuberculosis," Photoelectronics, vol.16, pp. 133-139, June 2007.

24. S.H. Guminetskiy, O.G. Ushenko, I.P. Polyanskiy, A.V. Motrych, F.V. Grynchuk, "The optical method for investigation of the peritonitis progressing process," Proceedings of the SPIE, vol. 7008, Article ID 700827, April 2008.

25. Alexander Ushenko, Sergej Yermolenko, Alexander Prydij, Stepan Guminetsky, Ion Gruia,

(C) 2011, V. Lashkaryov Institute of Semiconductor Physics, National Academy of Sciences of Ukraine 
Ovidiu Toma, Konstantin Vladychenko, “Statistical and fractal approaches in laser polarimetry diagnostics of the cancer prostate tissues," Proceedings of the SPIE, vol. 7008, Article ID 70082C, April 2008.

26. A.G. Ushenko, A.I. Fediv, Yu.F. Marchuk, "Correlation and fractal structure of Jones matrices of human bile secret," Proceedings of the SPIE, vol. 7368, Article ID 73681Q, July 2009.

27. A.G. Ushenko, Yu.Ya. Tomka, V.I. Istratiy, "Polarization selection of two-dimensional phaseinhomogeneous birefringence images of biotissues," Proceedings of the SPIE, vol. 7388, Article ID 73881L, December 2009.

28. A.G. Ushenko, A.I. Fediv, Yu.F. Marchuk, "Singular structure of polarization images of bile secret in diagnostics of human physiological state," Proceedings of the SPIE, vol. 7368, Article ID 73681S, July 2009.
29. S.B. Yermolenko, A.G. Ushenko, P. Ivashko, "Spectropolarimetry of cancer change of biotissues," Proceedings of the SPIE, vol. 7388, Article ID 73881D, December 2009.

30. A.G. Ushenko, I. Z.Misevich, V. Istratiy, I. Bachyns'ka, A. P. Peresunko, Omar Kamal Numan, and T. G. Moiysuk, "Evolution of Statistic Moments of 2D-Distributions of Biological Liquid Crystal Net Mueller Matrix Elements in the Process of Their Birefringent Structure Changes,” Advances in Optical Technologies, vol. 2010, Article ID 423145, March 2010.

31. O. V. Dubolazov, A. G. Ushenko, V. T. Bachynsky, A. P. Peresunko, and O. Ya. Vanchulyak, "On the Feasibilities of Using the Wavelet Analysis of Mueller Matrix Images of Biological Crystals,” Advances in Optical Technologies, vol. 2010, Article ID 162832, March 2010. 